\title{
Glucocorticoids in breast cancer treatment: real benefit or selection bias?
}

\author{
Gurkan Guner $^{1}\left[\mathbb{C}^{\circ} \cdot\right.$ Omer Dizdar $^{1}$
}

Received: 11 June 2018 / Accepted: 28 June 2018 / Published online: 26 July 2018

(c) Springer Science+Business Media, LLC, part of Springer Nature 2018

We read with great interest the article by Lin et al. published in Breast Cancer Research and Treatment on the effect of glucocorticoid (GC) use on survival in patients with breast cancer [1]. The authors showed that concomitant use of glucocorticoids improved survival in patients who received adjuvant anthracycline-based chemotherapy for stage I-III breast cancer. The authors suggested that low doses of GC might have a priming effect and potentiate the cytotoxic effect of chemotherapy on tumor cells or the immunomodulatory effects of short-term use of low doses of GC might be the underlying mechanism for the improved survival. Although these potential mechanisms may explain the findings, we have an additional concern that should be addressed in the discussion.

The association between toxicity of chemotherapy and clinical outcomes has long been recognized regarding cancer treatments. Abola et al abstracted treatment toxicity and clinical outcome data from a sample of 99 phase III oncology randomized clinical trials and showed that treatments with relatively greater toxicity compared with their controls had relatively higher rates of clinical efficacy in terms of progression-free survival (PFS). In particular, greater relative gastrointestinal toxicity among treatments was associated with greater relative PFS compared with controls $(P=0.007)$ [2]. Among patients with locally advanced breast cancer treated with neoadjuvant FAC regimen (5- fluorouracil, adriamycin, cyclophosphamide), Chintamani et al showed a significant positive correlation between the presence of vomiting $(r=+0.558)$, alopecia $(r=+0.802)$, and response

This letter to the editor refers to the article available at doi: https ://doi.org/10.1007/s10549-018-4787-x and a rebuttal letter to this letter to the editor is available at doi: https://doi.org/10.1007/s1054 9-018-4872-1.

Gurkan Guner

gunergurkan@yahoo.com

1 Division of Medical Oncology, Department of Internal Medicine, Hacettepe University Faculty of Medicine, Hacettepe University, Sihhiye, 06100 Ankara, Turkey to neoadjuvant chemotherapy. A significant negative correlation was observed between the absence of side effects and poor response to neoadjuvant chemotherapy [3]. The study of Ching-Hung et al. was a retrospective study and GC use was not randomized but GCs were ordered by the physicians on an as-needed basis. Thus we can assume that the patients who received more GC were those who experienced more GC-treated toxicities including nausea/vomiting, hypersensitivity reactions, etc., and needed additional or higher doses of GC for the treatment of these toxicities. Given the association between toxicity and efficacy in the abovementioned studies, one explanation for the improved efficacy in this patient population might be simply selection bias, i.e., patients with higher GC use might represent a subgroup who experienced higher toxicity and improved efficacy. Considering the toxicities of GCs, potential benefit of GCs on overall survival should be prospectively confirmed before implementing in clinical practice.

Funding No funding was received.

\section{Compliance with ethical standards}

Conflict of interest All authors declared that they have no competing interest.

\section{References}

1. Lin CH, Chuang PY, You SL, Chiang CJ, Huang CS, Wang MY, Chao M, Lu YS, Cheng AL, Tang CH (2018) Effect of glucocorticoid use on survival in patients with stage I-III breast cancer. Breast Cancer Res Treat. https://doi.org/10.1007/s1054 9-018-4787-x

2. Abola MV, Prasad V, Jena AB (2014) Association between treatment toxicity and outcomes in oncology clinical trials. Ann Oncol 25:2284-2289. https://doi.org/10.1093/annonc/mdu444

3. Chintamani SV, Singh JP, Lyall A, Saxena S, Bansal A (2004) Is drug-induced toxicity a good predictor of response to neoadjuvant chemotherapy in patients with breast cancer?-a prospective clinical study. BMC Cancer 4:48. https://doi. org/10.1186/1471-2407-4-48 